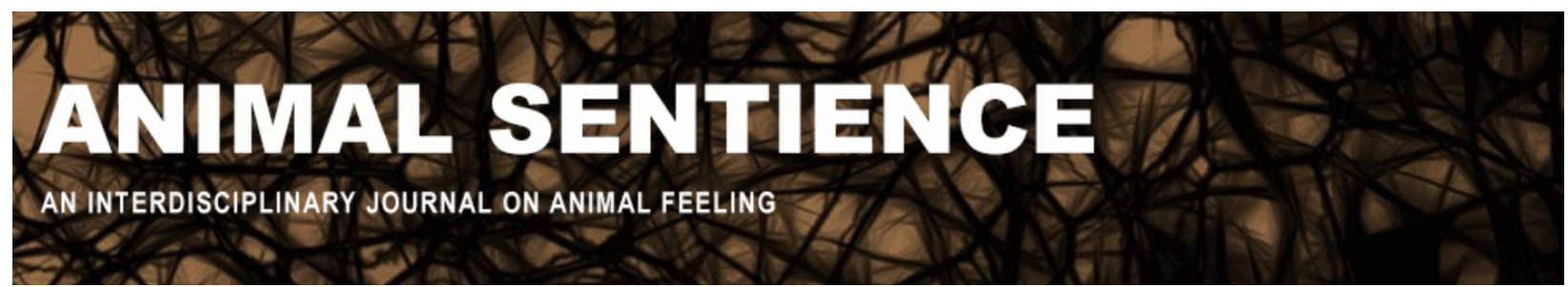

Mikhalevich, Irina (2017) Consciousness, evidence, and moral standing. Animal Sentience 13(2)

DOI: $10.51291 / 2377-7478.1208$

Date of submission: 2017-07-01

Date of acceptance: 2017-07-11

(c)

This article has appeared in the journal Animal

Sentience, a peer-reviewed journal on animal

cognition and feeling. It has been made open access,

free for all, by WellBeing International and deposited

in the WBI Studies Repository. For more information,

please contact

wbisr-info@wellbeingintl.org.

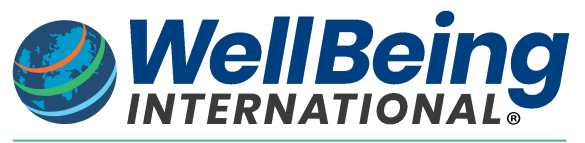

SOLUTIONS FOR PEOPLE, ANIMALS AND ENVIRONMENT 


\title{
Consciousness, evidence, and moral standing
}

Commentary on Woodruff on Teleost Consciousness

\author{
Irina Mikhalevich \\ Department of Philosophy \\ Rochester Institute of Technology
}

\begin{abstract}
Woodruff (2017) claims to have identified the neural correlates of phenomenal consciousness ("p-consciousness") in fishes, and argues that these neurological data, along with behavioral evidence, suggest that teleost fishes are in all probability sentient organisms. Woodruff's case may be strengthened by challenging key assumptions behind a common criticism of accounts such as his: that fishes cannot be $p$-conscious because they lack the cortical structures necessary for $p$-consciousness. A more serious objection to Woodruff's proposal would be that his evidence for p-consciousness establishes only that fishes are "access-conscious" ("aconscious"), where a-conscious states are cognitive representations that are made available to cognitive processing. This criticism calls into question his inference from certain cognitive states to the presence of $p$-consciousness, and may have significant implications for the moral standing of fishes and their treatment in fish welfare policy, since $p$-consciousness, but not a-consciousness alone, is typically thought to ground moral standing. I conclude that this criticism does not raise a decisive objection to Woodruff's argument, or to its usefulness for ethics and policy.
\end{abstract}

Irina Mikhalevich, Assistant Professor at the Department of Philosophy at Rochester Institute of Technology, does research on the philosophy of animal cognition, which lies at the intersection of the philosophy of science, philosophy of mind, philosophy of biology, and value theory.

https://philosophy.artsci.wustl.edu/people/irina-mikhalevich

\section{The cortex: Searching for homology in all the wrong places}

According to Woodruff (2017), although fish lack the cortical structures associated with mammalian phenomenal consciousness ( $p$-consciousness), regions of the teleost fish pallium and optic tectum can accommodate many of the functions associated with consciousness in mammals. For instance, the optic tectum contributes to such functions as sensory integration and binding, selective attention, and the segregation of the sensory modalities, while regions of the pallium may contribute to (inter alia) regulating motivational and affective states. These neuronal connections and anatomy are, he argues, "complex enough to support the reentrant computational processes proposed to underlie sentience in mammals." In addition to these structural features of the teleost tectum, the pallium contains similar types of neurophysiological features, such as EEG signatures and neurotransmitter systems, suggesting that affective and motivational states modulate behavioral response in fishes in much the way they do in mammals. Woodruff concludes that this neurobiological data, combined with behavioral data suggestive of 
consciousness, provides strong evidence against the view that a cortex or homologous structure is required for consciousness, and for the presence of $p$-consciousness in teleost fishes.

While I leave it to others to assess the merits of Woodruff's specific neurobiological claims, I believe that he gives more weight to the objection that fishes cannot be conscious because consciousness requires either a cortex or a homologous structure (which fish lack), than this objection deserves. Not only does this claim (a) beg the question against the potential multiple realizability of consciousness (Jones 2016), but also, by requiring homology at the level of gross morphology, it (b) takes a simplistic approach to homology in cognition and its underlying substrates and (c) neglects the possibility that consciousness arose numerous times independently in the vertebrate clade (Allen 2013). There is no reason to assume a priori that consciousness can only be realized in mammals (let alone animals in general) by the specific structures that give rise to it in humans; there are many a posteriori (empirical) reasons to reject the cortex-centered view of consciousness (Karten 2015).

Evidence that consciousness can be realized by noncortical structures of the brain comes from the behavioral and neurobiological study of birds, such as corvids (crows, ravens, jays) and psittacines (parrots). These animals are well-known for their flexible behavioral repertoires and cognitive sophistication, despite lacking a recognizable structure that is homologous with the mammalian cortex. Until relatively recently, their behavioral flexibility had been explained away as arising from simple cognitive mechanisms because their brains were thought to be incapable of sustaining complex cognition. As behavioral evidence mounted, however, neuroscientists began to search for structures in the avian brain that could do the work of the mammalian cortex - in effect, for a "functional" avian cortex. This search led researchers to the avian telencephalon, which is not homologous with the mammalian cortex at the level of gross morphology, but appears to be homologous at lower levels (e.g., microcircuitry, biochemistry, and cell-type) (Olkowicz et al. 2016). It is plausible that homology at lower levels can underwrite consciousness in morphologically divergent but functionally similar macroscopic neuroanatomies in disparate groups.

We cannot, therefore, rule out the possibility that evolution has converged on $p$ consciousness numerous times, realized by a diversity of structures and neurophysiological features at both the level of gross morphology and at the lower levels. Indeed, recent developments in invertebrate neurobiology point in the direction of remarkable neurobiological diversity underpinning consciousness across the animal kingdom. There is mounting behavioral evidence of complex cognition and perhaps consciousness in cephalopod mollusks such as the octopus and cuttlefish, insects such as bumblebees, and arachnids such as jumping spiders (Edelman \& Seth 2009; Barron \& Klein 2016). For instance, Klein \& Barron (2016) maintain that insect brains are functional analogues of regions of vertebrate brains that they take to support experiential (phenomenal) consciousness. ${ }^{1}$

If fish, cephalopods, and some insects are indeed p-conscious, then basic forms of consciousness may be evolutionarily ancient, extending as far back in time as the Cambrian. This view is supported by the hypothesis that consciousness emerged as an adaptive response to the

\footnotetext{
1 "While there is no obvious similarity in the gross anatomy ... there is an overall similarity in functional architecture between the vertebrate midbrain [which $K \& B$ take to support $p$-consciousness in vertebrates] and the insect brain as a whole" (Klein \& Barron 2016, p. 5).
} 
information-processing challenges of active lifestyles, such as the need to transform dynamic multisensory perceptual inputs into a coherent and temporally stable scene as distinct from the organism's own movements (the "re-afference problem") and to coordinate and control complex bodies; and to identify relevant environmental cues. All of these may contribute to the "effective decision-making" needed for adaptive action (Mikhalevich, Powell, \& Logan 2017; Powell in prep.). These problems are efficiently addressed by perceptual and cognitive abilities typically correlated with consciousness, such as sensory binding and directed attention. ${ }^{2}$ Whether consciousness emerged multiple times in evolutionary history, or whether it evolved once long ago, the lesson for the cortex-centered view is the same: consciousness is unlikely to be unique to mammals or to require mammalian neural correlates of consciousness. As Braithwaite \& Droege (2016) write, "the question is not whether fish have a cortex or an insula, but whether they have the functional capacity for [indicators of consciousness such as] global integration and attentional amplification."

\section{P-consciousness, a-consciousness, and moral standing}

A more serious objection to Woodruff's analysis is that even if he has successfully established that fish behavior and neurobiology indicate a capacity for global integration and sensory binding, these capacities are only indicative of $a$-consciousness and not $p$-consciousness. ${ }^{3}$ Call this the "awithout-p" objection. ${ }^{4}$ The separability of a-consciousness and $p$-consciousness is difficult to deny as a matter of conceptual possibility. The question is whether we have reason to think that these different sorts of consciousness come apart in the actual world.

If the a-without-p objection holds, then Woodruff's account may be of limited ethical import because not all forms of consciousness matter morally. The distinction between access and p-consciousness has important implications for the class of beings who are recognized as having "moral standing," or interests that are valuable for the entity's own sake. Forms of consciousness that matter morally are those that give rise to interests, which in turn ground rights and corresponding obligations toward the interest-bearers (Kahane \& Savulescu 2009). For example, beings for whom pains, joys, and the satisfactions of desires feel like something have interests vis-à-vis those pains, joys, and satisfactions. These "experiential" interests associated

\footnotetext{
2 "For active animals with well-developed spatial senses, it is computationally more effective to resolve the reafference problem once in a unified sensory model than to resolve it in a dispersed and peripheral way for each sense independently" (Klein \& Barron 2016, p. 3).

${ }^{3}$ Woodruff adopts the view that "mental images are part of primary, sensory consciousness and, therefore, contribute to "something it is like." Since fishes have the perceptual systems necessary for mental image formation, he concludes that they also possess the substrates necessary for $p$-consciousness. However, it is possible that mental images may be $p$-unconscious, such as scenes viewed through the display screen of an iPhone. While mental images are surely importantly different from images displayed on a cell phone, the point here is that visual scenes may be transformed into representations without those representations rising to the level of conscious.

${ }^{4}$ The a-without-p objection accepts what the philosopher of cognitive science Chalmers (1995) calls the "hard problem" of consciousness, or the causal-explanatory gap between the mechanistic properties of the brain as reflected (e.g.) in a-consciousness, and the experiential quality of p-consciousness. It bears noting, however, that although Block's distinction between a-consciousness and p-consciousness has been largely accepted in the philosophical community since its introduction in 1995, it is not uncontroversial.
} 
with feeling, wanting, and acting beings are the basis of moral consideration. By contrast, there is nothing that it is like to be a merely a-conscious animal and, thus, the content of such an animal's life cannot matter to the animal. A-conscious beings lacking $\mathrm{p}$-consciousness thus fail to satisfy a crucial precondition of moral standing (Jaworska \& Tannenbaum 2013; Butler \& Cotterill 2006). Moreover, while other forms of consciousness (e.g., self-consciousness) or cognition may generate additional interests for $p$-conscious beings, $p$-consciousness seems to be a necessary condition for moral standing as such. ${ }^{5}$

Has Woodruff succeeded in showing that fishes are not merely a-conscious, thus overcoming the a-without-p objection? I suggest that not only is he entitled to the claim that fishes are likely p-conscious, but that ethicists would be justified in drawing on his analysis in defending the moral standing of fishes. This is because the objection is predicated on an unjustified standard of evidence. Much of the animal ethics literature appears implicitly committed to the idea that certain abilities explicable in exclusively a-conscious terms nevertheless give rise to moral standing. These include sophisticated cognitive states (e.g., mirror-self recognition, tool use, mindreading), affective states (e.g., fear or pain), and perceptual abilities (e.g., perceptual binding). If some a-conscious states ground moral standing, then either (i) they are presumed to be attended by p-consciousness; or (ii) they ground moral standing on their own. A third possibility is that none of these abilities can in principle indicate $p$ consciousness. This third, deflationary option is frequently taken to be the default or "null" position, and thus the burden of proof is often placed on hypotheses that presume to show that a given animal is p-conscious. As a consequence, accounts such as Woodruff's are required to demonstrate their explanatory or predictive superiority over the "null."6

Yet the case for the deflationary view is at best equivocal. The empirical arguments in its favor draw largely on outlier cases from human lesion studies (e.g., with blindsight patients) and from examples of subconscious human behavior (e.g., driving without attending to the road), of which the relevance to intact and attentive animals is contested. Against this, evidence of $p$ consciousness reliably co-occurring with a-consciousness comes from experimentally controlled first-person reports combined with neurological signatures. If such reports are valid in the human case, they ought to be valid in the nonhuman case as well (Edelman \& Seth 2009), as thinking otherwise would be biologically unparsimonious (Butler \& Cotterill 2006). It would be a mistake to stake a case for the burden of proof on such equivocal evidence. Woodruff's case for $p$ consciousness in fishes is, therefore, not undermined by the possibility that the behavior and neurobiology he describes can be explained by reference to a-consciousness alone.

That said, philosophers of mind may find Woodruff's account of consciousness in fish unsatisfying, preferring a higher standard of evidence before granting $p$-consciousness to animals. However, for both animal cognition scientists with working hypotheses in hand, and for ethicists who should err on the side of attributing morally relevant properties in the absence of conclusive evidence, Woodruff's case for $p$-consciousness in fish ought to suffice. ${ }^{7}$

\footnotetext{
${ }^{5}$ Even for the Kantian, p-consciousness appears be a necessary condition for moral standing.

${ }^{6}$ A parallel situation exists in comparative cognition research, wherein the "null" position is frequently that an animal lacks some cognitive feature (Mikhalevich 2016).

${ }^{7}$ Indeed, variations in standards of evidence across disciplines are both expected and arguably justified by the different epistemic and practical goals of these disciplines (Block 2011).
} 


\section{Ethics as evidence}

Finally, it is worth noting an additional, subtler, ethical dimension to the science of consciousness: the ethical implications of theories of $p$-consciousness may implicitly bias our estimates of the plausibility of those theories. Scientists and philosophers alike may find a theory of consciousness less intuitively plausible if it appears to imply that we have moral obligations toward beings who have typically been excluded from the moral community. However, if a new theory of consciousness implies that, for example, molecules or thermostats have moral standing, then rather than conclude that the attribution of consciousness is problematic because consciousness has such and such ethical implications, we should conclude that our theory of moral standing is inadequate. Similarly, some consciousness researchers may question the plausibility of the idea that fishes or insects are p-conscious because the implication - that we have ethical obligations toward these organisms - may strike them as absurd. Indeed, the ethical implication may seem so implausible that it might function as an informal reductio ad absurdum of the claim that there is something that it's like to be a cichlid, a jumping spider, or a honeybee. However, while rejecting a normative theory for its apparently absurd ethical implications may be rational, it is fallacious to reject a descriptive scientific theory for its normative implications. There may be good scientific reasons for rejecting a theory of consciousness that is so permissive that even molecules qualify; but distaste for a proposition does not alter its truth-value. In short, we must not allow our moral intuitions to prejudice the plausibility estimates of analyses such as Woodruff's, even (or especially) when these analyses ascribe consciousness to animals as alien in morphology and lifeways as fishes and invertebrates.

\section{References}

Allen, C. (2013) Fish cognition and consciousness. Journal of Agricultural and Environmental Ethics 26.1: 25-39.

Barron, A. B., and Klein, C. (2016) What insects can tell us about the origins of consciousness. Proceedings of the National Academy of Sciences 113.18: 4900-4908.

Block, N. (2011) Perceptual consciousness overflows cognitive access. Trends in Cognitive Sciences 15.12: 567-575.

Braithwaite, V. A., and Droege, P. (2016) Why human pain can't tell us whether fish feel pain. Animal Sentience 3(3).

Butler, A. B., and Cotterill, R. M. J. (2006) Mammalian and avian neuroanatomy and the question of consciousness in birds. Biological Bulletin 211.2: 106-127.

Chalmers, D. (1995) Facing up to the problem of consciousness. Journal of Consciousness Studies 2.3: 200-219.

Edelman, D. B., and Seth, A. K. (2009) Animal consciousness: a synthetic approach. Trends in Neurosciences 32.9: 476-484.

Jaworska, A., and Tannenbaum, J. (2013) The Grounds of Moral Status, Stanford Encyclopedia of Philosophy (Summer 2013 Edition), E. N. Zalta (Ed.).

Jones, R. C. (2016) Fish sentience and the precautionary principle. Animal Sentience 3(10).

Kahane, G., and Savulescu, J. (2009) Brain damage and the moral significance of 
consciousness. Journal of Medicine and Philosophy 34.1: 6-26.

Karten, H. J. (2015) Vertebrate brains and evolutionary connectomics: On the origins of the mammalian 'neocortex'. Philosophical Transactions of the Royal Society B 370.1684: 20150060.

Klein, C., and Barron, A. B. (2016) Insects have the capacity for subjective experience. Animal Sentience 9(1).

Mikhalevich, I. (2015) Experiment and animal minds: Why the choice of the null hypothesis matters. Philosophy of Science 82.5: 1059-1069.

Mikhalevich, I., Powell, R., and Logan, C. (2017) Is behavioural flexibility evidence of cognitive complexity? How evolution can inform comparative cognition. Interface Focus 7.3: 20160121.

Olkowicz, S., Kocourek, M., Lučan, R. K., Porteš, M., Tecumseh Fitch, W., Herculano-Houzel, S., and Němec, P. (2016) Birds have primate-like numbers of neurons in the forebrain. Proceedings of the National Academy of Sciences: 201517131.

Powell, R. (in prep.). Contingency and Convergence in the History of Life (MIT Press).

Woodruff, M. (2017) Consciousness in teleosts: There is something it feels like to be a fish. Animal Sentience 13(1). 